\title{
Ocular tracking of biological and nonbiological motion: The effect of instructed agency
}

\author{
Jan Zwickel • Mathias Hegele • Marc Grosjean
}

Published online: 20 December 2011

(C) Psychonomic Society, Inc. 2011

\begin{abstract}
Recent findings suggest that visuomotor performance is modulated by people's beliefs about the agency (e.g., animate vs. inanimate) behind the events they perceive. This study investigated the effect of instructed agency on ocular tracking of point-light motions with biological and nonbiological velocity profiles. The motions followed either a relatively simple (ellipse) or a more complex (scribble) trajectory, and agency was manipulated by informing the participants that the motions they saw were either human or computer generated. In line with previous findings, tracking performance was better for biological than for nonbiological motions, and this effect was particularly pronounced for the simpler (elliptical) motions. The biological advantage was also larger for the human than for the computer instruction condition, but only for a measure that captured the predictive component of smooth pursuit. These results suggest that ocular tracking is influenced by the internal forward model people choose to adopt.
\end{abstract}

J. Zwickel

Department Psychology, Ludwig Maximilians University, Munich, Germany

M. Hegele

Neuromotor Behavior Lab, Department of Sport Science, Justus Liebig University,

Giessen, Germany

M. Grosjean

Leibniz Research Centre for Working

Environment and Human Factors,

Dortmund, Germany

J. Zwickel ( $₫)$

Neuro-Cognitive Psychology, Department of Psychology, Ludwig Maximilians University Munich,

Leopoldstrasse 13,

80802 Munich, Germany

e-mail: Jan.Zwickel@psy.lmu.de
Keywords Ocular tracking - Smooth pursuit · Saccadic eye movement $\cdot$ Biological motion · Instructed agency

It is well recognized that ocular tracking of a moving object involves predictive mechanisms in both monkeys (Keller \& Johnsen, 1990) and humans (Rashbass, 1961; van den Berg, 1988; Wexler \& Klam, 2001). For example, the predictive component of smooth pursuit is susceptible to expectations derived from verbal or visual cues regarding the upcoming motion trajectory (Kowler, 1989). More generally, it has been argued that smooth pursuit can actually be considered as a concatenation of initiating saccades, which, because of their predictive nature, introduces an anticipatory (saccadic) component in ocular tracking (Barnes, Barnes, \& Chakraborti, 2000; see also Kao \& Morrow, 1994). We sought to understand how this component is affected when people are asked to track biological and nonbiological point-light motions while being led to believe that the motions were produced by an animate agent or not.

Initially, investigating hand movements, Viviani and colleagues showed that individuals are unable to move in a fashion that violates the two-thirds power law, even after extensive training under visual (Viviani \& Mounoud, 1990) or kinesthetic (Viviani, Baud-Bovy, \& Redolfi, 1997) guidance. This law captures a functionally significant covariation between the curvature and the tangential velocity of voluntary hand movements. While drawing an ellipse, for example, one typically slows down as curvature increases and speeds up as curvature decreases (see Fig. 1a, c). The name of the law stems from the fact that the tangential velocity of such movements is a function of the radius of curvature raised to the power $1-\beta$, where $\beta$ typically has a value of 2/3 (Lacquaniti, Terzuolo, \& Viviani, 1983; Viviani \& Terzuolo, 1982). 
Of particular interest here, de'Sperati and Viviani (1997) found that eye movements also seem to conform to this principle. They asked participants to follow with their eyes a dot that moved along an ellipse with either a biological velocity profile (i.e., consistent with the two-thirds power law) or a nonbiological velocity profile (i.e., inconsistent with the law). Tracking performance was assessed by counting the number of saccades and what they called the saccade mismatch index (MI) and the smooth pursuit MI. The saccade MI captured the extent of saccadic compensation that was needed during ocular tracking and, thus, the effectiveness of smooth pursuit. The MI for smooth pursuit reflected how much distance between gaze and dot was tolerated before saccadic compensation occurred. Consistent with the law, all measures revealed better performance (i.e., lower values) for ocular tracking of biological motions.

Superior performance for biological motion profiles has been attributed to a reliance on internal motor prediction models (e.g., Saunier, Papaxanthis, Vargas, \& Pozzo, 2008). Such (forward) models are assumed to be based on people's actual action repertoire, and the predictions they make are thus "biological" by nature (e.g., Haruno, Wolpert, \& Kawato, 2001) - hence, the performance advantage for biological over nonbiological conditions. However, while earlier views stressed the importance of kinematic cues for selection of the prediction model, more recent findings also stress the role
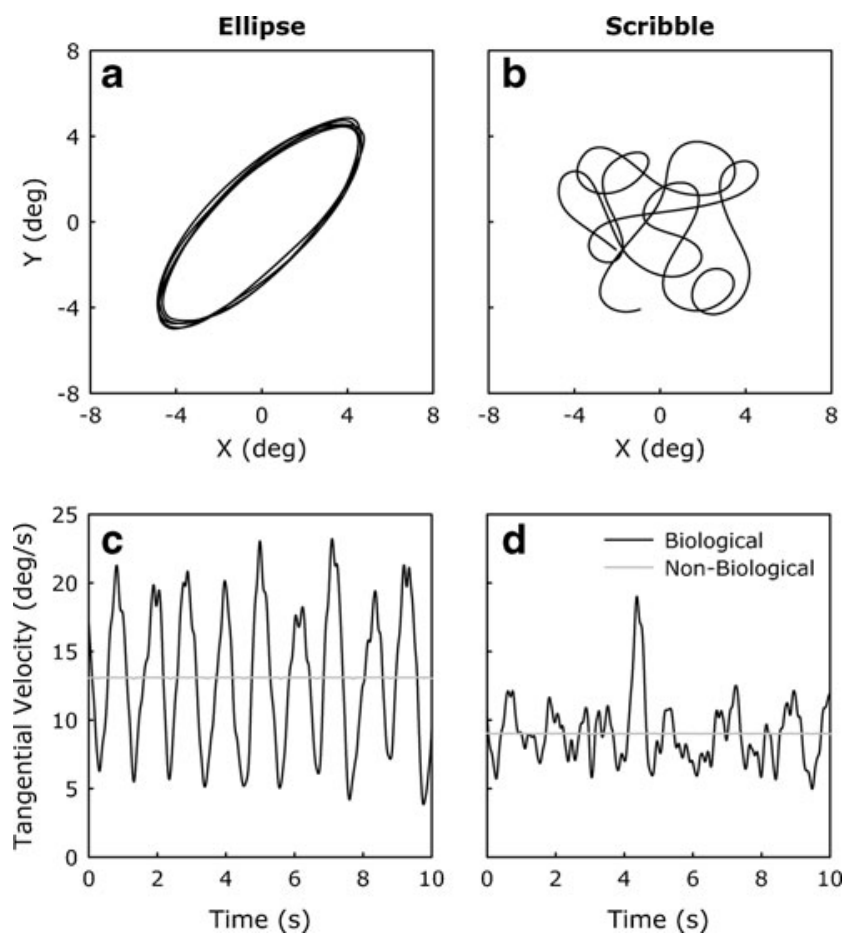

Fig. 1 Examples of the trajectories from the ellipse (a) and scribble (b) conditions. The corresponding tangential velocities as a function of time and velocity type (biological, nonbiological) are presented in the lower panels (c and d, respectively). Degrees refer to visual angles of the interpretational stance of the observer. In particular, systematic effects of agency manipulations have been reported at both behavioral (Stanley, Gowen, \& Miall, 2007) and neurophysiological (Stanley, Gowen, \& Miall, 2010; Wheatley, Milleville, \& Martin, 2007) levels, suggesting that at least some kind of strategic control can be exerted over the selection of motor prediction models in such situations.

For example, relying on a task introduced by Kilner, Paulignan, and Blakemore (2003), Stanley et al. (2007) examined the execution of oscillatory linear arm movements while simultaneously observing a spatially in/congruent dot motion that had either a biological (accelerating and decelerating) or a nonbiological (constant) velocity profile. An advantage of congruent over incongruent conditions, in terms of less movement variability, was present for both velocity profiles when participants had been told that the dot motion represented prerecorded movements performed by humans. However, no interference was observed when the instructions designated the same motion as computer generated. This suggests that agency attribution indeed modulates how what we perceive interacts with how we move. Consistent with this idea, it has been shown that agency instructions not only affect behavioral judgments regarding the presence of human motion in scrambled point-light displays, but also modulate neural activity, particularly in the paracingulate cortex, in response to otherwise identical visual stimuli (Stanley et al., 2010).

To investigate the effects of instructed agency on ocular tracking, we asked participants, as in de'Sperati and Viviani (1997), to track the motion of a dot that had either a biological or a nonbiological velocity profile. Instructed agency was manipulated by informing the participants that the to-be-tracked motion either was a prerecorded movement of a human or was computer generated. As was alluded to above, the perceptuomotor system's compliance with the two-thirds power law should lead to better tracking of biological than of nonbiological motions. This benefit of tracking biological motions should be observed independently of instruction condition. However, tracking performance should also be affected by conceptual knowledge about the motion (i.e., human vs. computer generated) that should allow participants to select a prediction model that could or could not match the actual kinematics of the dot.

In addition to using ellipses, scribble trajectories were included (see Fig. 1b, d). The two-thirds power law has been shown to hold for these more complex movements as well (Lacquaniti, Terzuolo, \& Viviani, 1984), and they were added to test less stereotypical motions than previously employed. On the basis of the generality of the law, one could expect the benefit of biological over nonbiological motions to be present for scribbles as well, even if overall performance may decrease due to the complexity of these motions. However, efficient visuomotor tracking relies on 
anticipating both the velocity and spatial trajectory of the motions to be tracked. Thus, the benefit of tracking biological motions may be apparent only when the motion trajectory is predictable as well, as is the case for ellipses but not for scribbles.

\section{Method}

\section{Participants}

Eighteen individuals (mean age $=24.0$ years; range $=19-27$ years; 2 males; 1 left-handed) were paid for their participation. All participants had normal or corrected-to-normal vision and were unaware of the purpose of the study.

\section{Apparatus and stimuli}

Monocular eye tracking was performed with the Eyelink 1000 in combination with the Tower Mount chin-and-head rest (SR Research Ltd., Ontario, Canada). By default, the left eye was tracked; however, when a better calibration could be achieved with the right eye, the tracked eye was switched. Viewing distance was fixed at $55 \mathrm{~cm}$, and eye movement data were sampled at $1000 \mathrm{~Hz}$ with a resolution of $0.01^{\circ}$. At the beginning of each trial, it was checked whether participants fixated a cross with a precision of at least $0.5^{\circ}$ of visual angle. If participants failed to do so, a recalibration was started. The stimuli were presented on a color CRT monitor with the refresh rate set at $100 \mathrm{~Hz}$, and the experiment was controlled with the Experiment Builder software (SR Research Ltd., Ontario, Canada).

Stimuli consisted of motions of a black dot (diameter $=$ $0.79^{\circ}$ ) that moved on a white background without leaving a trace for $\sim 10 \mathrm{~s}$. The motion of the dot depicted either an ellipse or a scribble that spanned an area of approximately $7^{\circ} \times 7^{\circ}$. Examples of the two trajectory types are presented in Fig. 1a, b. Each trajectory was based on previously recorded movements of one of the authors that had been made on an Ultrapad A3E graphics tablet (Wacom Co. Ltd., Japan) that sampled the $x$ and $y$ positions of a hand-held stylus at $70 \mathrm{~Hz}$. For the ellipses, a paper template was placed on the tablet to guide the movements. The main semiaxis of the ellipses was always rotated by $45^{\circ}$ relative to horizontal, and three templates were used with varying aspect ratios between the minor and major axes $(0.25,0.35$, and 0.45$)$. The latter manipulation was included to approximate the variations in size present in the scribbles. Five movement recordings were performed for each template, resulting in 15 ellipse trajectories. Fifteen different scribble trajectories were created by changing directions of the stylus smoothly and, to the degree possible, randomly over the 10 -s recording interval.
For the biological velocity conditions, each original trajectory was resampled at the refresh rate of the monitor (i.e., $100 \mathrm{~Hz}$ ) by relying on piecewise cubic spline interpolation. The corresponding tangential velocity profiles can be found in Fig. 1c, d. To create the nonbiological velocity conditions, each original trajectory was initially resampled at $10000 \mathrm{~Hz}$, using the method described above, and then resampled again at $100 \mathrm{~Hz}$ by selecting approximately equidistant samples. This resulted in nonbiological tangential velocities that were essentially constant and that equaled the average tangential velocity of the original (biological) movement (see Fig. 1c, d).

\section{Design and procedure}

There were two blocks of 60 trials $(=2$ velocity types [biological, nonbiological] $\times 2$ trajectory types [ellipse, scribble] $\times 15$ exemplars) in which every possible combination of conditions was presented once. The blocks differed only with respect to the instruction given to the participants regarding the origin of the dot motions. In the "human" instruction condition, they were informed that they would see traces of a person's arm movements depicted by a dot. In the "computer" instruction condition, they were told that they would be presented with computergenerated dot motions. The instructions were provided in written form before each block, and the order of the instructions was counterbalanced across participants.

Each trial started with the presentation of the dot at the first position of the to-be-shown trajectory. The motion began after participants had fixated the dot for $300 \mathrm{~ms}$ by maintaining their gaze within a small circular area (diameter $=0.94^{\circ}$ ) centered around it. As soon as the dot started to move, the task of the participants was to follow it with their eyes as accurately as possible. The motion terminated 9,980 ms later, at which point the display went blank and a new trial started. The eyetracker was calibrated prior to each block and whenever participants failed to fixate the dot within $3,000 \mathrm{~ms}$ at the beginning of a trial. To familiarize participants with the task, the first block was preceded by five practice trials that were excluded from the analysis.

\section{Data analysis}

Saccades were extracted using the Eyelink Parser (SR Research Ltd., Ontario, Canada) and were defined as eye movements that had a velocity or acceleration that exceeded $22 \%$ or $4000 \% \mathrm{~s}^{2}$, respectively. The velocity threshold was increased by the average velocity during the last $40 \mathrm{~ms}$ of pursuit (see the Eyelink manual for details).

Following de'Sperati and Viviani (1997), three measures were computed to assess ocular tracking performance for each participant and condition: number of saccades, mean 
saccade MI, and mean smooth pursuit MI. All measures were calculated over the entire trial duration and were then summed/averaged across the 15 exemplars for each trajectory type. Number of saccades simply referred to the total number of saccades that were made during a trial. Saccade MI was calculated by subtracting the distance (in visual angle) between gaze and dot after each saccade from the distance between gaze and dot at the beginning of the saccade. Smooth pursuit MI was calculated by subtracting the distance (again in visual angle) between gaze and dot at the end of saccade $n$ from the distance between gaze and dot at the beginning of saccade $n+1$.

To exclude outliers, absolute saccade and smooth pursuit MI values were discarded when they were greater than $1.89^{\circ}$ (50 pixels) or more than 3 standard deviations away from the mean of the respective measure. On average, this led to the exclusion of $4.3 \%$ of the MI data. Finally, separate three-way repeated measures analyses of variance (ANOVAs) were performed on the three performance measures, with instruction type (human, computer), velocity type (biological, nonbiological), and trajectory type (ellipse, scribble) as within-subjects factors.

\section{Results}

Before we turn to the dependent variables, Fig. 2a, b illustrates the relationship between radius of curvature and tangential velocity for the ellipses and scribbles, respectively. These scatterplots are similar to those reported by de'Sperati and Viviani (1997), and the $\beta$ s for smooth pursuit (pursuit) are close to the expected value of $2 / 3$ (ellipse, $\beta=1-$ slope $=1-$ $0.36=0.64$; scribble, $\beta=1-0.35=0.65)$. What can also be seen from these examples is that the data for dot motion (target) and smooth pursuit overlap more and have more similar slopes for ellipses than for scribbles.

As can be seen in Fig. $3 a-c$, the means of the three performance measures were all lower for ellipses than for scribbles and for biological than for nonbiological velocities. This general pattern was corroborated by the ANOVAs, which yielded significant main effects of trajectory type [number of saccades, $F(1,17)=245.82, \eta_{\mathrm{p}}{ }^{2}=.94$, $p<.001$; saccade MI, $F(1,17)=401.91, \eta_{\mathrm{p}}{ }^{2}=.96, p<.001$; smooth pursuit MI, $\left.F(1,17)=320.11, \eta_{\mathrm{p}}{ }^{2}=.95, p<.001\right]$ and velocity type [number of saccades, $F(1,17)=57.26, \eta_{\mathrm{p}}{ }^{2}=$ $.77, p<.001$; saccade MI, $F(1,17)=45.11, \eta_{\mathrm{p}}{ }^{2}=.73, p<.001$; smooth pursuit MI, $\left.F(1,17)=47.62, \eta_{\mathrm{p}}{ }^{2}=.74, p<.001\right]$.

However, the advantage of biological over nonbiological velocities was particularly pronounced for tracking of elliptical trajectories. Consistent with this observation, the interaction between trajectory type and velocity type was significant for the two MIs [saccade MI, $F(1,17)=11.80$, $\eta_{\mathrm{p}}{ }^{2}=.41, p<.01$; smooth pursuit MI, $F(1,17)=22.10$,

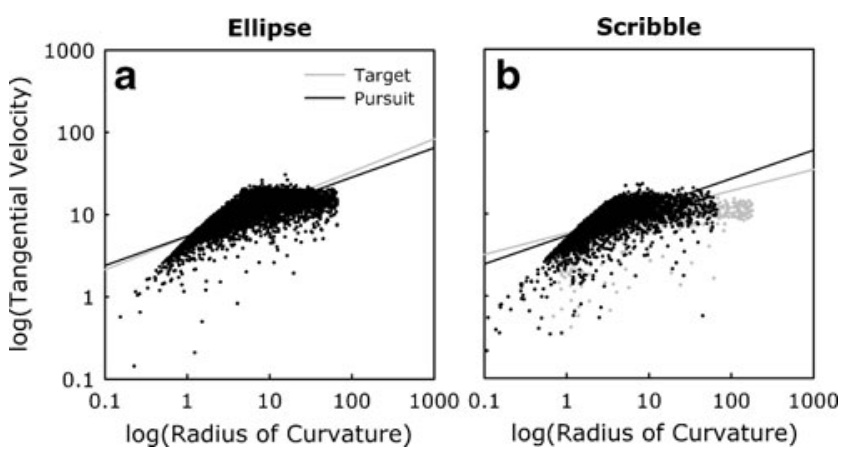

Fig. 2 Examples of the relationship between radius of curvature and tangential velocity for dot motion (Target) and smooth pursuit (Pursuit). The values are presented in logarithmic scales, and regression lines are also included. Curvature was estimated using a 3-point algorithm that calculated the radius of an imaginary circle that would go through those points. Both examples are taken from the human instruction conditions with biological velocity profiles for ellipses (a) and scribbles (b)

$\left.\eta_{\mathrm{p}}{ }^{2}=.57, p<.001\right]$ and just missed significance for number of saccades, $F(1,17)=4.15, \eta_{\mathrm{p}}{ }^{2}=.20, p=.057$. Separate follow-up ANOVAs on the MIs for each trajectory type revealed significant simple main effects of velocity type for ellipses [saccade MI, $F(1,17)=32.81, \eta_{\mathrm{p}}{ }^{2}=.66, p<.001$; smooth pursuit $\left.\mathrm{MI}, F(1,17)=50.65, \eta_{\mathrm{p}}{ }^{2}=.75, p<.001\right]$, but not for scribbles (both $p \mathrm{~s}>.08$ ).

Perhaps more interestingly, there was also a significant instruction type $\times$ velocity type interaction for the saccade MI, $F(1,17)=6.83, \eta_{\mathrm{p}}{ }^{2}=.29, p<.05$, but not for the other two measures (both $p \mathrm{~s}>.50$ ). However, neither of the simple main effects of instruction type for each velocity type was significant (both $p \mathrm{~s}>.60$ ). This interaction therefore reflects the fact that the benefit of biological over nonbiological motions was larger for the human than for the computer instruction condition (see Fig. 3b).

None of the other main effects or interactions were significant (all $\mathrm{ps}>.09){ }^{1}$

\section{Discussion}

The present results replicate the advantage of ocular tracking an elliptic motion with a biological, as compared

\footnotetext{
${ }^{1}$ The results considered until now were collapsed across instruction order (human first, computer first). To determine whether instruction order may have affected the results, we included it as a betweensubjects factor in the ANOVA on the critical measure of saccade MI. The only additional effect to reach significance was an instruction order $\times$ instruction type interaction, $F(1,16)=6.44, \eta_{\mathrm{p}}{ }^{2}=.29, p<.05$ (all other $p \mathrm{~s}>.14$ ): Participants who began with the computer instruction performed better with the human instruction, and vice versa for participants who began with the human instruction. Since there was only one block per instruction type, this pattern merely reflects a within-experiment learning effect in that performance was, on average, higher in the second block of the experiment.
} 
Fig. 3 Mean number of saccades (a), mean saccade mismatch index (MI; b), and mean smooth pursuit MI (c) as a function of instruction type (human, computer), velocity type (bio[logical], non-bio[logical]), and trajectory type (ellipse, scribble).

Degrees refer to visual angles, and error bars correspond to

2 times the standard error that was obtained by pooling the error terms of the relevant repeated measures ANOVA

(Loftus \& Masson, 1994)
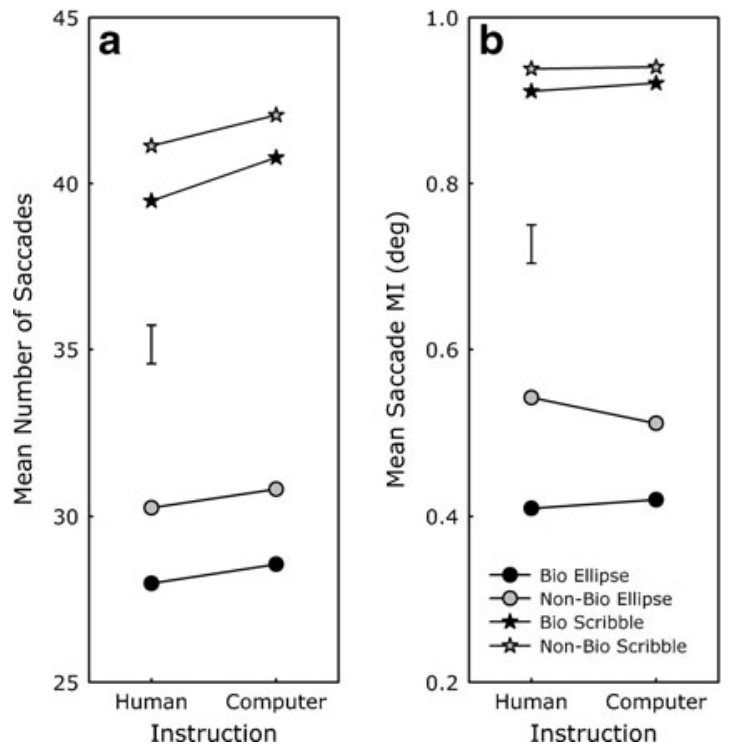

with a nonbiological, velocity profile. This advantage was originally reported by de'Sperati and Viviani (1997), and, due to the generality of the two-thirds power law, we expected it to generalize to more complex motions as well. However, this advantage was, at least statistically, restricted to simple (elliptic) motions; no difference between the two types of velocity profiles was found with more complex (scribble) motions. Thus, one interpretation of the present findings is that biological velocity profiles lead to better tracking performance only when the motions are cyclical and contain few directional changes. That is, the benefit for biological velocity profiles may be observed only when the spatial trajectory of the motion can be anticipated as well. This was the case for ellipses, but not for scribbles. Closer scrutiny, however, reveals that there was a systematic, albeit nonsignificant, trend indicating that biological velocity profiles allowed for better tracking of scribbles as well. Accordingly, we cannot rule out the possibility that the present study may have lacked the statistical power to establish such an effect.

As has already been alluded to, one explanation for the advantage of biological over nonbiological velocity profiles is the reliance on internal motor prediction models that are "biological" by nature (e.g., Haruno et al., 2001; Saunier et al., 2008). Interestingly, we found that instructed agency modulated the size of this advantage. In particular, it was larger for the human than for the computer instruction, but only for the more predictive component of ocular tracking (i.e., saccade MI). We believe this finding reflects how people select a predictive model to successfully guide their eye movements. Indeed, selection of a model can be based on two sources of information: online signals relating to motion perception and offline knowledge about the origin of an observed motion (cf. Haruno et al., 2001; Imamizu et al., 2007; Wolpert \& Kawato, 1998). Both sources of information can bias model selection by assigning high probabilities to certain predictive models.

Returning to the critical interaction for saccade MI, we believe that the human instruction led participants to assign high probabilities to biological prediction models. However, when confronted with a nonbiological velocity profile, the assigned probability required correction based on online sensory information. This should result in better/worse (predictive) performance for biological/nonbiological velocity profiles. Similarly, one could assume that the computer instruction would lead to an opposite effect. However, the latter was perhaps smaller or nonexistent because of a predominance of "biological" prediction models in humans. Thus, the effect of instructed agency should be stronger for the human condition.

This interpretation is further supported by the findings of Stanley and colleagues (2007): The interference of observed motions on movement production was present in their human but not in their computer instruction condition. For the present data, only the human instruction may have affected performance by incurring a somewhat larger "cost" for nonbiological motions. Although one might expect the effect of instructed agency to be larger for motions that are less easily tracked (i.e., scribbles), this was not the case in the present study. However, in line with a point made earlier, the ability to predict the velocity of an observed motion may affect performance only when one can also anticipate its spatial trajectory, which was more difficult in the case of scribbles.

To summarize, we extend existing findings by demonstrating an influence of instructed agency on eye movements, and we do so with a measure that is tailored to capture predictive components of processing. This provides additional support for the notion of an independent contribution of predictive components in smooth pursuit (Rashbass, 1961). 
Our results are also consistent with those of Stanley et al. (2007), which suggest that the selection of internal forward models can be biased by verbal instructions regarding the origin of an observed motion. Therefore, ocular tracking seems to be penetrable by cognitive concepts.

Author note We would like to thank Wilfried Kunde for sharing code to collect data from the graphics tablet and two anonymous reviewers for helpful comments. This work was supported by German Research Foundation (DFG) Grant EC 142, Excellence Cluster "Cognition for Technical Systems (CoTeSys)."

\section{References}

Barnes, G. R., Barnes, D. M., \& Chakraborti, S. R. (2000). Ocular pursuit responses to repeated, single cycle sinusoids reveal behavior compatible with predictive pursuit. Journal of Neurophysiology, 84, 2340-2355.

de'Sperati, C., \& Viviani, P. (1997). The relationship between curvature and velocity in two-dimensional smooth pursuit eye movements. Journal of Neuroscience, 17, 3932-3945.

Haruno, M., Wolpert, D., \& Kawato, M. (2001). MOSAIC model for sensorimotor learning and control. Neural Computation, 13, 2201-2220.

Imamizu, H., Sugimoto, N., Osu, R., Tsutsui, K., Wada, Y., \& Kawato, M. (2007). Explicit contextual information selectively contributes to predictive switching of internal models. Experimental Brain Research, 181, 395-408.

Kao, G. W., \& Morrow, M. J. (1994). The relationship of anticipatory smooth eye movement to smooth pursuit initiation. Vision Research, 34, 3027-3036.

Keller, E. L., \& Johnsen, S. D. S. (1990). Velocity prediction in corrective saccades during smooth pursuit eye movements in monkey. Experimental Brain Research, 80, 525-531.

Kilner, J. M., Paulignan, Y., \& Blakemore, S.-J. (2003). An interference effect of observed biological movement on action. Current Biology, $13,522-525$

Kowler, E. (1989). Cognitive expectations, not habits, control anticipatory smooth oculomotor pursuit. Vision Research, 9, 1049-1057.
Lacquaniti, F., Terzuolo, C. A., \& Viviani, P. (1983). The law relating kinematic and figural aspects of drawing movements. Acta Psychologica, 54, 115-130.

Lacquaniti, F., Terzuolo, C. A., \& Viviani, P. (1984). Global metric properties and preparatory processes in drawing movements. In S. Kornblum \& J. Requin (Eds.), Preparatory states and processes (pp. 357-370). Hillsdale: Erlbaum.

Loftus, G. R., \& Masson, M. E. J. (1994). Using confidence intervals in within-subjects designs. Psychonomic Bulletin \& Review, 1, 476-490.

Rashbass, C. (1961). The relationship between saccadic and smooth tracking eye movements. The Journal of Physiology, 159, 326-338.

Saunier, G., Papaxanthis, C., Vargas, C. D., \& Pozzo, T. (2008). Inference of complex human motion requires internal models of action: Behavioral evidence. Experimental Brain Research, 185, 399-409.

Stanley, J., Gowen, E., \& Miall, R. C. (2007). Effects of agency on movement interference during observation of a moving dot stimulus. Journal of Experimental Psychology: Human Perception and Performance, 33, 915-926.

Stanley, J., Gowen, E., \& Miall, R. C. (2010). How instructions modify perception: An fMRI study investigating brain areas involved in attributing human agency. NeuroImage, 52, 389-400.

van den Berg, A. V. (1988). Human smooth pursuit during transient perturbations of predictable target movement. Experimental Brain Research, 72, 95-108.

Viviani, P., Baud-Bovy, G., \& Redolfi, M. (1997). Perceiving and tracking kinesthetic stimuli: Further evidence of motor-perceptual interactions. Journal of Experimental Psychology: Human Perception and Performance, 23, 1232-1252.

Viviani, P., \& Mounoud, P. (1990). Perceptuo-motor compatibility in pursuit tracking of two-dimensional movements. Journal of Motor Behavior, 22, 407-443.

Viviani, P., \& Terzuolo, C. A. (1982). Trajectory determines movement dynamics. Neuroscience, 7, 431-437.

Wexler, M., \& Klam, F. (2001). Movement prediction and movement production. Journal of Experimental Psychology: Human Perception and Performance, 27, 48-64.

Wheatley, T., Milleville, S. C., \& Martin, A. (2007). Understanding animate agents: Distinct roles for the social network and mirror system. Psychological Science, 18, 469-474.

Wolpert, D., \& Kawato, M. (1998). Multiple paired forward and inverse models for motor control. Neural Networks, 11, 13171329. 